\title{
DIAGNOSTIC ROLE OF ENDOBRONCHIAL WASHINGS AND BIOPSY IN SMEAR NEGATIVE PULMONARY TUBERCULOSIS AND ITS CORRELATION WITH HIGH RESOLUTION COMPUTED TOMOGRAPHY CHEST FINDINGS
}

\author{
Maryam Hussain, Sultan Mehmood Kamran, Rizwana Azam, Asifullah Khan*, Sohaib Ahmed, Usman Bin Ali** \\ Pak Emirates Military Hospital/National University of Medical Sciences (NUMS) Rawalpindi Pakistan, *Combined Military Hospital/National University of \\ Medical Sciences (NUMS) Rawalpindi Pakistan, ${ }^{* *}$ Combined Military Hospital Lahore/National University of Medical Sciences (NUMS) Pakistan
}

\begin{abstract}
Objective: To determine role of endobronchial washings/biopsy in diagnosis of smear negative pulmonary tuberculosis and evaluate their association with computed tomography findings.

Study Design: Cross-sectional - analytical study.

Place and Duration of Study: Department of Pulmonology, Pak Emirates Military Hospital, Rawalpindi Pakistan, from Jan to Jun 2020.

Methodology: Patients having symptoms of pulmonary tuberculosis and abnormal findings on chest imaging with negative sputum smears for acid fast bacilli were selected for endobronchial washings. Bronchoscopy for washings was carried out in these patients and sample was sent for Acid fast bacilli stain, Mycobacterium tuberculosis gene Xpert and acid fast bacilli culture and sensitivity bacterial culture and fungal hyphae. In case of mass lung endobronchial and/or transbronchial lung biopsy was done and sample was sent for histopathology Active pulmonary tuberculosis was labelled as per definition.

Results: Out of 105, 75 (71.4\%) were male and 30 (28.6\%) were female. Mean age was $55.69 \pm 17.33$ years. Total 40 (38.09\%) were diagnosed with pulmonary tuberculosis (based on smear, genexpert, culture and histopathology). In these 40 patients, computed tomographic findings showed consolidation in $10(25 \%)$ cases, cavity in $7(17.5 \%)$, bronchiectasis in $3(7.5 \%)$, mass in $1(2.5 \%)$, pleural effusion in $2(5 \%)$, lymphadenopathy in $1(2.5 \%)$ and tree-in-budappearance in $12(30 \%)$ cases.

Conclusion: High resolution computed tomography and endobronchial washing both combined together increase the diagnostic yield in smear negative cases.
\end{abstract}

Keywords: Endobronchial washings, High-resolution computed tomography, Pulmonary tuberculosis, Smear negative.

This is an Open Access article distributed under the terms of the Creative Commons Attribution License (https://creativecommons.org/licenses/by-nc/4.0/), which permits unrestricted use, distribution, and reproduction in any medium, provided the original work is properly cited.

\section{INTRODUCTION}

Pulmonary tuberculosis remains a major cause of death in developing countries with 1.5 million deaths in 2018. According to WHO statistics for tuberculosis in 2018, Pakistan ranked $5^{\text {th }}$ among the high-burden countries globally, with fourth largest prevalence of multi-drug resistant TB worldwide ${ }^{1}$. According to WHO global TB report 2018, the incidence of TB in Pakistan is $267 / 100,000$ population. Among the many factors that have caused the emergence of drug-resistant $\mathrm{TB}$, it is primarily appropriated to delays in diagnosis of tuberculosis, in addition to inappropriate drug therapies ${ }^{2}$.

Diagnosis of pulmonary tuberculosis relies on bacteriological examination that includes sputum smear microscopy and culture however, the diagnostic yield of these tests is low with sensitivity ranging from $25-45 \%{ }^{3}$. There is a good proportion of patients of pulmonary tuberculosis who are smear negative that is, either they don't expectorate or are sputum negative

Correspondence: Dr Maryam Hussain, Classified Medical Specialist, Pak Emirates Military Hospital, Rawalpindi Pakistan

Received: 29 Sep 2020; revised received: 09 Apr 2021; accepted: 22 Apr 2021 for AFB. The choices in these cases is either to begin empirical ATT based on clinico-radiological findings, which has its own side effects or to perform an invasive test like bronchoscopy for endobronchial sampling4. The sensitivity of HRCT in cases of smear positive pulmonary tuberculosis is as high as $96 \%$. Due to low bacillary load, typical HRCT features in sputum smear negative cases of TB are not observed ${ }^{5}$.

Bronchial washings in smear negative casescan be tested through multiple techniques such as smear, microscopy, MTB Gene Xpert, cytology, culture and sensitivity ${ }^{6}$. According to British Thoracic Society guidelines, bronchoscopy is a safe procedure, the complication rate is low, around $1.1 \%$ in tertiary centres with good standard of care. Being a minimally invasive procedure, it is valuable in the diagnosis of smear negative pulmonary TB and to provide an alternate diagnosis ${ }^{7}$.

This rationale of this study was to determine role of endobronchial washings in diagnosis of smear negative pulmonary tuberculosis, and evaluate their correlation with HRCT findings, as incorporating all available diagnostic tools to detect the TB cases at earlier stages can decrease spread in community and help in long-term survival. 


\section{METHODOLOGY}

This was analytical cross-sectional study carried out at the Pulmonology department of Pak-Emirates Military Hospital Rawalpindi, from January to June 2020. Permission from hospital ethics committee (A/ 28/EC/200/2020) was sought and informed consent was taken from the patients who participated in the study. Sample size was calculated using RaoSoft with sample population of 105 . Non-probability sampling was used and a total of 105 cases were studied who were advised High resolution computed tomography of chest based on subacute and chronic respiratory tract symptoms. Patients were taken from both in patient and out patient departments. All of them, had either no sputum or were smear negative for AFB. Carefully excluding comorbids and other lung diseases like ILD, CA lung and any contraindications for bronchoscopy, smear positive cases and previously treated pulmonary TB cases were excluded. After taking informed consent, patients undergoing bronchoscopy were first administered topicalanaesthesia in the form of $2 \%$ lignocaine gel. $4 \%$ lignocaine in normal saline gargles were used to topically anaesthetize pharyngeal region. A $2 \mathrm{~mm}$ bronchoscope was inserted through the nose and 30cc sample was collected from the affected lung segment based on HRCT findings. Patients with a detected mass on HRCT additionally underwent biopsy along with EBW. The samplesobtained were sent for AFB smear, MTB GeneXpert, culture and sensitivity for Mycobacterium tuberculosis. Bacterial culture and sensitivity and fungal hyphae. Cytology and histopathology in cases ofendobronchial and transbronchial lung biopsy were sent for alternative diagnosis. Cepheid GeneXpert was done as per standard instructions. Culture medium used was MGIT/BACTEC. Positive case of Tuberculosis was labelled as any patient with positive MTB GeneXpert or positive acid-fast bacilli in culture and sensitivity. Data was collected through records kept at the Bronchoscopy Department at PakEmirates Military Hospital. The HRCT findings of patients were studied. Findings on HRCT included mass (>30 mm of hyper dense region), cavitation (hypodense area within pulmonary consolidation, mass or nodule), consolidation, bronchiectasis, pleural effusion, lymphadenopathy and tree-in-bud appearance. Data was analysed using SPSS-20. For categorical data, descriptive statistics were used. The proportion of males and females within the selected sample was calculated, as was the mean age of the patients. Mean and standard deviation of all findings were calculated. Frequencies and percentages were calculated for categories.

\section{RESULTS}

A total of 105 patients who underwent bronchoscopy and whose complete data was available on follow-up, were included in this study. Out of these 75 $(71.4 \%)$ were males and $30(28.6 \%)$ were females. The mean age was $55.69 \pm 17.33$ years.

On bronchoscopy, based on analysis of bronchoalveolar washings and biopsy results, $40(38.09 \%)$ were found to have pulmonary tuberculosis. In these 40 patients, high resolution computed tomographic findings showed consolidation in $10(25 \%)$ cases, cavity in $7(17.5 \%)$, bronchiectasis in $3(7.5 \%)$, mass in 1 $(2.5 \%)$, pleural effusion in $2(5.0 \%)$, lymphadenopathy in $1(2.5 \%)$ and tree-in-bud appearance in $12(30 \%)$ cases (table-II). Out of these 40 confirmed cases, 7 had features of endobronchial TB on bronchoscopy with findings of endobronchial mucosal irregularity, erythyma, edema and/or bronchial stenosis. These cases with endobronchial abnormalities had findings of tree in bud with or without consolidations on HRCT chest. 3 patients out of these 7, with mucosal biopsy showed caseating granuloma consistent with tuberculosis. Rest were MTB positive on bronchial washings from the affected area on CT.

On HRCT chest, 11 patients had mass lung, out of which 6 underwent endobronchial luminal mass

Table-I: Diagnostic yield in patients undergoing bronchoscopy $(n=105)$.

\begin{tabular}{l|c|c}
\hline Diagnostic Yield & Frequency & Percentage \\
\hline Pulmonary tuberculosis & 40 & 38.09 \\
\hline Nondiagnostic & 35 & 33.33 \\
\hline Carcinoma lung & 6 & 5.71 \\
\hline $\begin{array}{l}\text { Nontuberculous } \\
\text { mycobacteria }\end{array}$ & 8 & 7.6 \\
\hline Fungal hyphae & 4 & 3.80 \\
\hline $\begin{array}{l}\text { Chroniccaseating } \\
\text { granuloma }\end{array}$ & 4 & 3.80 \\
\hline Pseudomonas & 5 & 4.76 \\
\hline Gram positive cocci & 7 & 6.66 \\
\hline
\end{tabular}

Table-II: High resolution computed tomographic findings of patients diagnosed with tuberculosis $(n=40)$.

\begin{tabular}{l|c|c}
\hline $\begin{array}{l}\text { High resolution computed } \\
\text { tomographic findings }\end{array}$ & Frequency & Percentage \\
\hline Consolidation & 10 & 25 \\
\hline Soft-tissue nodules & 4 & 10 \\
\hline Cavity & 7 & 17.5 \\
\hline Bronchiectasis & 3 & 7.5 \\
\hline Mass & 1 & 2.5 \\
\hline Effusion & 2 & 5.0 \\
\hline Lymphadenopathy & 1 & 2.5 \\
\hline Tree-in-bud appearance & 12 & 30 \\
\hline
\end{tabular}


biopsy and 5 patients underwent transbronchial lung biopsy. Bronchogenic carcinoma was diagnosed in 6 $(5.71 \%)$ patients with the help of endobronchial mass biopsy findings. Out of these cases, on histopathology, $3(50 \%)$ patients were diagnosed as a case of squamous cell carcinoma, 2 (33.33\%) had adenocarcinoma, 1 $(16.66 \%)$ was diagnosed as small cell carcinoma. Findings on HRCT in these patients were mass lung with or without soft tissue nodules. Only 1 patient with transbronchial biopsy had caseating granuloma on histopathology.

Out of the remaining cases, nontuberculous mycobacteria (NTM) were detected in $8(7.6 \%)$ patients based on AFB seen on smear and negative MTB DNA on GENE xpert test. Fungal hyphae were seen in endobronchial washings of 4 (3.8\%) patients. All of above had cavities and/or bronchiectasis, tree in bud appearance on HRCT. Pseudomonas was grown in 5 (4.76\%) patients and gram positive cocci are seen on microscopy of endobronchial washings in $7(6.6 \%)$ patients however their culture was negative. Thirty five (29.5\%) patients had normal microscopy, culture of endobronchial washings and nonsuggestive histopathology on biopsy and further investigations were advised.

\section{DISCUSSION}

Pulmonary tuberculosis can mimic a lot of diseases in clinicoradiological findings, reliability on sputum has limitations as sputum may be false negative if the disease is mild, paucibacillary or the patient is giving saliva instead of sputum. Sputum smear can find AFB in $50-60 \%$ of cases 8 . Rates of detection are lower in resource poor countries like ours and delayed diagnosis can lead to spread of disease. Culture which is gold standard can take several weeks and the clinicians face the difficult decision of either starting empirical treatment or to wait for the culture results. Cultureis both expensive and labour intensive process. The ideal test with maximum sensitivity in our set up as recommended by WHO is Gene Xpert test on spu-tum which gives fastest result but again in sputum naïve or smear negative cases the yield is lower. Bronchoscopic washings for AFB in centres where bronchoscopy is available can increase the diagnostic yield.

This study has described the role of endobronchial washings and biopsy in diagnosis and the common patterns of findings on HRCT in smear negative patients diagnosed with tuberculosis. Our study found that endobronchial washings are superior to HRCT in accurate diagnosis of pulmonary tuberculosis. In cases which were diagnosed as TB on the basis of endobr- onchial washings, a wide spectrum of HRCT findings was noted that included consolidation, cavitation, bronchiectasis, mass, pleural effusion, lymphadenopathy and tree-in-budappearance.

In a previous study done in the same centre on HRCT findings in sputum smear positive patients, tree-in-bud appearance was reported in $68 \%$ of the cases, whereas, in our study it was reported in $30 \%$ of cases which highlights the differences in radiological findings between smear positive and smear negative cases $^{9}$. In most patients, multiple findings were observed, therefore, no single feature on HRCT was solely found to be diagnostic of tuberculosis. Diagnosis based on radiological findings can be non-specific and requires confirmation by endobronchial washings. In study by Kosaka and his colleagues in 2005, they found that air space consolidation, cavitation and ground glass opacities occurred significantly morefrequently in the smear positive than in the smear negative active PTB patients while the frequency of micronodules did not differ between the two groups ${ }^{10}$. On theother hand in the study of Lee et al, the sensitivity, specificity, positive predictive value and positive likelihood ratioof HRCT in the diagnosis of smear negative pulmonary tuberculosis were $80 \%, 70 \%, 71 \%$ and $2.71 \%$. They concluded that HRCT alone had relatively good sensitivity but the low positive predictive value hampered the decision of starting anti-TB medication ${ }^{11}$. A study on 100 patients with smear negative tuberculosis in Birjand university of Medical Sciences Iran concluded that radiological findings for pulmonary TB cannot be used to diagnose but are rather suggestive ${ }^{12}$.

The main role of HRCT for diagnosing pulmonary TB istheselection of probable or highly suspected pulmonary TB with pulmonary infiltrates of unknown origin and with negative sputum smears for further laboratory tests or bronchoscopy .

Our study revealed such smear negative patients with nonspecific findings on HRCT, whose bronchoscopy showed features of mucosal irregularity, edema, necrosis and bronchial stenosis. Three out of 7 such patients who underwent endobronchial mucosal biopsy in addition to endobronchial washings showed caseating granuloma on histopathology results, consistent with diagnosis of tuberculosis. In a review article by Shehzad from Agha Khan University Hospital, bronchial biopsy is the most valuable sampling method forestablishing early diagnosis in endobronchial tuberculosis with a diagnostic yield of $30-84 \%^{13}$. 
Bronchoscopy was also useful in making alternative diagnosis like in our study non tuberculous mycobacterium, fungal hyphae, pseudomonas and gram positive cocci were found in non tuberculous cases who had computed tomographic findings suggestive of pulmonary TB.

In a prospective study done by Schoch et al on 101 asylum seekers in Switzerl and with radiological findings suggestive of active tuberculosis, it was concluded that radiology findings are a poor guide to initiation of anti tuberculous treatment. The study compared the diagnostic yield between a regular, induced sputum and endobronchial washings. The results showed a significantly higher yield in smear negative cases with bronchial washings ${ }^{14}$. Similarly in a study conducted on 120 suspected cases of pulmonary tuberculosis with negative smear in DOW teaching hospital by Faisal et al concluded that bronchial washings Gene Xpert yielded diagnosis in $69.2 \%{ }^{15}$. Also in a study from india on 75 suspected smear negative patients, bronchial washings smear for AFB and histopathology made immediate diagnosis possible in $48.33 \%{ }^{16}$.

However, in a resource-poor healthcare setup such as Pakistan, the cost effectiveness of endobronchial washings inbronchoscopy procedures as a primary diagnostic tool is a point of concern. In a study conducted in India to evaluate the usefulness and costeffectiveness of bronchial washings in addition to endobronchial biopsies, the addition of bronchial washing was found to be helpful in diagnosis butwas not cost-effective ${ }^{17}$. Furthermore, bronchoscopy facilities are only availablein specialized tertiary care centres in Pakistan and are not readily accessible. In this study we have not considered the yield of tuberculosis in induced sputum which is a more cost effective method and has proved to have better yield than regular sputum. In a study by Luo et al, on 586 patients sputum induction had almost similar sensitivity and specificity compared to bronchoscopic specimens in smear negative tuberculosis ${ }^{18}$.

A few limitations of this observational study were small sample size, risk of selection bias. There was an absence of uniform analysis as all endobronchial samples were not analysed by a single pathologist. The results of endobronchial washings were not compared with induced sputum sample analysis.

\section{CONCLUSION}

High-resolution computed tomography scan provides a set of non-specific findings that require complementary evidence for diagnosis in cases of smear negative pulmonary tuberculosis. Bronchial washings are a more reliable diagnostic tool with higher sensitivity and specificity of results. Both tests combined together increase the diagnostic yield, While the wider applicability of endobronchial procedures will increase diagnostic yield, for a developing country with economic limitations it might not prove to be cost-effective.

\section{CONFLICT OF INTEREST}

This study has no conflict of interest to be declared by any author.

\section{REFERENCES}

1. Global tuberculosis report 2018. Geneva: World Health Organization; 2018. Licence: CC BY-NC-SA 3.0 IGO. [Internet] Available at: http://apps.who.int/iris.

2. Abubakar I, Zignol M, Falzon D, Raviglione M, Ditiu L, Masham S, et al Drug-resistant tuberculosis: time for visionary political leadership. Lancet Infect Dis 2013; 13(6): 529-39.

3. Patel D, Kamdar DJ. Sputum versus bronchial washings for diagnosis of pulmonary tuberculosis- a prospective study of 53 patients. Int J Res Med Sci 2017; 5(4): 1266-69.

4. Raniga S, Parikh N, Arora A, Vaghani M, Vora PA, Vaidya V. Is HRCT reliable in determining disease activity in pulmonary tuberculosis?. Ind J Radiol Imag 2006; 16(1): 221-28.

5. Bhalla AS, Goyal A, Guleria R, Gupta AK. Chest tuberculosis: Radiological review and imaging recommendations. Ind J Radiol Imag 2015; 25(3): 213-25.

6. Shin JA, Chang YS, Kim TH, Kim HJ, Ahn CM, Byun MK. Fiberoptic bronchoscopy for the rapid diagnosis of smear-negative pulmonary tuberculosis. BMC Infect Dis 2012; 22(12): 141-45.

7. Du Rand IA, Blaikley J, Booton R, Chaudhuri N, Gupta V, Khalid $\mathrm{S}$, et al. British Thoracic Society guideline for diagnostic flexible bronchoscopy in adults: accredited by NICE. Thorax 2013; 68(Suppl-1): i1-44.

8. Campos LC, Vieira Rocha MV, Cunha Willers DM, Silva DR. Characteristics of patients with smear-negative pulmonary tuberculosis (TB) in a region with high TB and HIV prevalence. PLoS One 2016; 11(1): 1-8.

9. Naseem A, Saeed W, Khan S. High resolution computed tomographic patterns in adults with pulmonary tuberculosis. J Coll Phys Surg Pak 2008; 18(11): 703-7.

10. Kosaka N, Sakai T, Uematsu H, Kimura H, Hase M, Noguchi M, et al. Specific high-resolution computed tomography findings associated with sputum smear positive pulmonary tuberculosis. J Comput Assist Tomogr 2005; 29(6): 801-4.

11. Lee HM, Shin JW, Kim JY, Park IW, Choi BW, Choi JC, et al. HRCT and whole-blood interferon-c assay for the rapid diagnosis of smear-negative pulmonary tuberculosis. Respirat 2010; 79(2): 454-60.

12. Ebrahimzadeh A, Azarkar Z. Radiologic findings in patients with smear-negative pulmonary tuberculosis. Int J Mycobacteriol 2015; 4(1): 148-52.

13. Shahzad T, Irfan M. Endobronchial tuberculosis-a review. J Thorac Dis 2016; 8(12): 3797-2.

14. Schoch OD, Rieder P, Tueller C, Altpeter E, Zellweger JP, Rieder $\mathrm{HL}$, et al. Diagnostic yield of sputum, induced sputum, and bronchoscopy after radiologic tuberculosis screening. Am J Respir Crit Care Med 2007; 175(1): 80-86.

15. Faisal F. Role of bronchial washing Gene Xpert in smear negative cases of suspected pulmonary tuberculosis. Europ Respirat J 2018; 52(62): PA4769. 
16. Prakash P, Agarwal P, Gupta P, Gupta E, Dasgupta A. Comparison of induced sputum and broncholaveolar lavage fluid examination in the diagnosis of sputum negative pulmonary tuberculosis, Indian J Chest Dis Allied Sci 2016; 58(3): 173-5.

17. Gopathi NR, Mandava V, Namballa UR, Makala S. A Comparative study of induced sputum and bronchial washings in diagno- sing sputum smear negative pulmonary tuberculosis. J Clin Diagn Res 2016; 10(3): OC07-10.

18. Luo W, Lin Y, Li Z, Wang W, Shi Y. Comaprison of sputum induction and bronchoscopy in diagnosis of sputum smear-negative pulmonary tuberculosis: a systemic review and meta-analysis. BMC Pulm Med 2020; 20(1): 146-50. 